\title{
Luminescent probe based techniques for hypoxia imaging
}

\begin{abstract}
Hypoxia is a condition of tissue environments wherein a lower than normal level of oxygen is available, and it serves as the root cause and indicator of various diseases. Detection of hypoxia in tumors is imperative for furthering our understanding of the pathological effects and the development of proper treatments, as it is well established that hypoxic tumors are able to impede the cancer treatment process by being resistant to many therapies. It is important therefore to be able to detect hypoxia in tissues and tumors through in vivo imaging methods. A growing area for detection of hypoxia in vivo is the use of fluorescent/luminescent probes which has accelerated in recent years. The continued quest for improvements in selectivity and sensitivity has inspired researchers to pursue new strategies for fluorescence/luminescent probe design. This review will discuss various luminescent probes based on small molecules, dyes, macromolecules, and nanoparticles for sensitive and specific detection of oxygen levels directly or by indirect mechanisms such as the presence of enzymes or related factors that arise in a hypoxic environment. Following the particular mechanism of detection, each probe has specific structural and photophysical properties which permit its selectivity and sensitivity. These probes show promise in terms of low toxicity and high specificity among other merits discussed, and in providing new dimensions for hypoxia detection, these works contribute to future potential methods for clinical diagnosis of hypoxic tissues and tumors.
\end{abstract}

Keywords: Fluorescence, Imaging, Near-infrared, Hypoxia
Volume 6 Issue 3 - 2017

Sana Sandhu, LeNaiya Kydd, Justyn Jaworski
Department of Bioengineering, University of Texas at Arlington USA

Correspondence: Justyn Jaworski, Department of Bioengineering, the University of Texas at Arlington, 500 UTA Blvd., Arlington, TX 76019, USA, Tel 817-272-6778; Email justyn.jaworski@uta.edu

Received: October 27, 2017 | Published: November 20, 2017

\section{Introduction}

Hypoxia refers to an inadequate oxygen supply or low oxygen concentration and is the major cause of various diseases as oxygen plays a crucial role in many pathological and physiological processes in biological systems. ${ }^{1}$ Hypoxic conditions i.e. areas with $p \mathrm{O}_{2}$ values $\leq 2.5 \mathrm{mmHg}$ are known to be associated with solid tumors, stroke, cardiac ischemia, inflammatory diseases, ${ }^{2-5}$ vascular diseases like diabetic retinopathy and retinal vascular occlusion. ${ }^{6,7}$ Therefore, it is necessary to detect and monitor hypoxia in order to formulate proper therapeutic strategies to treat numerous diseases with which hypoxia is associated. Detection of hypoxia in solid tumors has been documented by a number of techniques including invasive immunostaining but also less-invasive techniques such as luminescence imaging, magnetic resonance imaging (MRI), and positron emission tomography (PET) among others..$^{8-15}$ Several exogenous and endogenous markers are also available for hypoxia evaluation in tumor microenvironments. ${ }^{14}$ For example, hypoxia is known to reduce the excess expression of reductive enzymes such as nitroreductase, azoreductase, and quinone reductase, among others, leading to reductive stress. ${ }^{16}$ Correspondingly, these enzymes act as viable markers providing an opportunity to detect hypoxia. Hypoxia makes solid tumors extremely difficult to treat since hypoxic tumor cells have a very limited blood supply; ${ }^{17}$ and consequently hypoxic tumors become resistant towards radiotherapy, chemotherapy, biotherapy, thermotherapy and anti-cancer drugs ${ }^{18}$ further causing problems in the treatment of cancer patients. Hypoxia may arise from inadequate blood flow in the cancerous tissues due to the vasculature abnormalities, from increased diffusion distances due to tumor expansion/geometries, or due to decreased oxygen transport capacity of the blood. ${ }^{18}$ As a result of such poor penetration, the treatment of hypoxic tumors with chemotherapy drugs leads to undesired side effects caused by non-selective drug accumulation in healthy tissues. ${ }^{19}$ Due to high resistance of hypoxic tumors to chemotherapy, radiotherapy, and other disadvantages, surgical treatment is typically suggested immediately after hypoxic tumor detection by imaging. ${ }^{20}$ Current limitations may lead to significant errors when patients are subjected to imaging for tumor diagnosis, prognosis and related therapeutic procedures. The diagnostic approaches using multimodality optical- and nuclear imaging based techniques for detection of brain tumor and tumor metastatic deposits in the brain could be a useful alternative. To overcome these barriers, non-invasive targeted methods such as peptide-based, nanoparticle coated delivery platforms ${ }^{21}$ have been developed to deliver targeted agents to tumors.

Optical methods, particularly fluorescent probes, have attracted attention since they offer reversible responses with no electrical interferences and do not destroy the analyte. Fluorescence probes are inexpensive and offer high spatiotemporal resolution as well as sensitivity. They also provide specific detection of target molecules with high signal-to-background ratios in complex environments such as cell lysates, living cells, and within living organisms. As a result, fluorescence imaging has been widely applied in biological detection with advantages of high resolution, adjustable emission wavelength, real-time monitoring and non-invasiveness. ${ }^{22}$ Such probes that have chromophores which exhibit absorption and emission in ultra-violet or visible range are inadequate for in vivo imaging. To utilize the potential of the fluorescent probes for improved detection of hypoxia for in vivo biological conditions, it is advantageous to move the development of fluorescent probes to the long-wavelength region (i.e., red and near-infrared). Near-infrared (NIR) optical methods provide a feasible opportunity for in vivo imaging due to many advantages such as deeper tissue penetration, minimum photodamage to biological samples, and minimum interference from background auto-fluorescence in living systems; thus, maximizing the specific signals from tissues and minimizing the background signals originating from off-target tissues. ${ }^{23}$ NIR fluorescent agents combined with fluorescence molecular tomography (FMT) have 
emerged as invaluable tools for quantitative, deep tissue imaging of a complex array of biological processes across a range of important areas of disease research including oncology, ${ }^{24}$ inflammation, ${ }^{25}$ angiogenesis ${ }^{26}$ and atherosclerosis. ${ }^{27}$ Hence, these optical probes have been widely used in non-invasive in vivo optical imaging for biomedical research following the development of advanced imaging probes in the NIR wavelength range where light signals are highly penetrable in biological tissues. ${ }^{28}$ These fluorescent probes can also undergo a number of chemical modifications including conjugation with effective therapeutic drugs to target cancer cells at different progressive stages. ${ }^{29}$

This review presents various fluorescent and luminescent molecular probes for detection of hypoxia following different mechanisms based on their structural properties. An overview of the precise probe formulations examined in this review is provided in Table 1. With these probe classes in mind, discussion is divided into three major parts based on the structural property of the molecules, specifically: dye or small molecule based probes; macromolecular/ chelator based probes; and inorganic nanoparticle based probes/ quantum dots. Within these sections, the probes are further examined with respect to direct versus indirect method of hypoxia detection. The hypoxia probes discussed below each undergo changes in their fluorescent properties due to the change in oxygen concentration or interaction with target hypoxia markers in the hypoxic regions. This fundamental property of hypoxia probes serves as the basis for effective fluorescence bioimaging for in vivo detection and hypoxic tumor monitoring in cancer cells for diagnostic purposes.

\section{Small molecule and dye based probes}

Several small or dye based fluorescent probes have been developed for hypoxia detection that follow detection mechanisms based on their structural and photophysical properties. Some of these probes detect oxygen levels directly while others determine hypoxia indirectly by the concentrations of hypoxia inducible factors. These inducible factors include nitroreductase, cytochrome $\mathrm{P} 450$ reductase, and carbonic anhydrase, which are abnormally expressed in hypoxic environment and cause an increase in reductive stress. ${ }^{16}$ Some of these probes contain specific moieties, such as azo groups, nitroimidazoles, $p$-nitro benzyls, nitro-furans, or azides, which are tethered to fluorophores as to quench their fluorescence emission and exhibit an "off-on" switching mechanism. After target analyte detection, fluorescence is regained by modulation of various processes like FRET (Förster resonance energy transfer), PIET (photoinduced electron transfer), ESIPT (excited state intramolecular proton transfer), ICT (internal charge transfer), self-assembly/disassembly, or chemical reactions producing free fluorescent groups which will be elaborated in the following section. In addition to fluorescent probes, phosphorescent probes, two-photon fluorescent probes, near infrared fluorescent (NIRF) probes, NIRF contrast agents containing tricarbocyanine dye, polymethine carbocyanine dyes and long wavelength fluorescence dyes with donor-acceptor properties will also be elaborated on for detection of hypoxia owing to their improved photophysical and biocompatibility properties as discussed below.

To begin, we will discuss several examples of the small or dye based probes which detect hypoxic conditions directly by estimating oxygen levels. For instance, probes MAR and MASR (1a) contain an azo-bond to a conjugated rhodamine derivatives as a scaffold fluorophore. As reported by K. Hanaoka et al these probes offer detection of hypoxia based on the photo switch concept resulting from the conformational change around the $\mathrm{N}=\mathrm{N}$ bond after photo-excitation. Under normal conditions MAR and MASR exhibited no fluorescence due to ultra-fast azo-bond rotation on photoexcitation whereas in hypoxia, reduction of MAR and MASR by reductases resulted in the generation of fluorescent rhodamine derivatives, 2Me RG or 2Me SiR600 (1b), respectively. This was accompanied by a 630 -fold or 20 -fold increase in fluorescence intensity of MAR or MASR, respectively, at a decreased oxygen concentration in hypoxic conditions. The excitation and emission wavelengths were 498 and $520 \mathrm{~nm}$ for MAR and 593 and $612 \mathrm{~nm}$ for MASR. Here, the direct conjugation of the azo bond to the conjugated system of the fluorophore provides the unique opportunity to tune the photochemical and fluorescence "off/on" switching of the hypoxia sensors. In addition, multicolor imaging with both probes allowed the visualization of oxygen concentration gradients in living cells, even allowing visualization of retinal hypoxia in a rat model of retinal artery occlusion by MAR. ${ }^{30}$
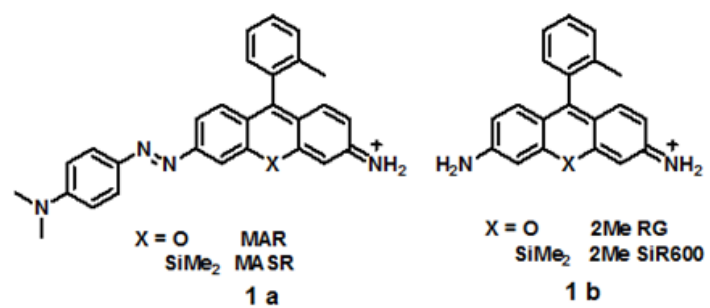

A series of azo-based iridium (III) "off-on" phosphorescence complexes have been designed and synthesized to detect hypoxia selectively. Non-phosphorescent probes Ir1-Ir8 (2a) on reduction under hypoxic conditions in an oxygen dependent process involving sequential electron transfer led to generation of high phosphorescence of the corresponding amines (2b). The phosphorescence intensity enhancement of 17.5-fold for Ir1, 16.9-fold for Ir2, 39.0-fold for Ir3, 38.4-fold for Ir4, 11.4-fold for Ir5, 13.5-fold for Ir6, 58.8-fold for Ir7, and 54.2-fold for Ir8 was observed. The maxima emission intensity ranged from $510 \mathrm{~nm}$ for Ir 1 and Ir 5 to $640 \mathrm{~nm}$ for Ir4 and Ir8, displaying green to red emissive solutions observable by the naked eye. Probes Ir1-Ir8 are eligible to function in a complex biological background because their phosphorescent response could be obtained in a mixed solution containing high concentration of ions or biorelated reducing agents in a suitable $\mathrm{pH}$ range. These probes could also penetrate 3D multicellular spheroids over $100 \mu \mathrm{m}$ and image the hypoxic regions in the center which are more suitable models for in vivo solid tumors using fluorescence imaging. ${ }^{31}$

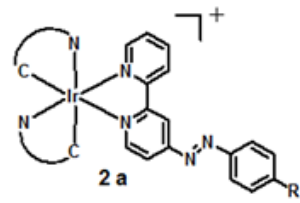

Ir1: $C \wedge N=d f p p y ; R=H$ Ir): $\mathrm{C} \wedge \mathrm{N}=\mathrm{ppy} ; \mathrm{R}=\mathrm{H}$ Ir $3: C \wedge N=2$ - $;$ q; $R=H$
Ir $4: C \wedge N==d b q ; R=H$ Ir5: $\mathrm{C} \wedge \mathrm{N}-\mathrm{dfppy} ; \mathrm{R}-\mathrm{N}\left(\mathrm{CH}_{3}\right)_{2}$
Ir6: $\mathrm{C} \wedge \mathrm{N}=\mathrm{ppy} ; \mathrm{R}=\mathrm{N}\left(\mathrm{CH}_{3}\right)_{2}$ Ir7: $\mathrm{C} \wedge \mathrm{N}-2 . \mathrm{pq} ; \mathrm{R}-\mathrm{N}\left(\mathrm{CH}_{3}\right)_{2}$ Ir8: $\mathrm{C} \wedge \mathrm{N}=\mathrm{dbq}: \mathrm{R}=\mathrm{N}\left(\mathrm{CH}_{3}\right)$

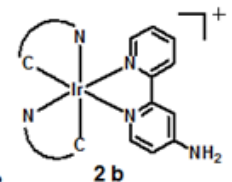
2 b $\mathrm{IrNH}_{2} 1: \mathrm{C} \wedge \mathrm{N}-\mathrm{dfppy}$ $\operatorname{lr}^{2} \mathrm{NH}_{2}: \mathrm{C}^{\wedge} \mathrm{N}=\mathrm{ppy}$
$\operatorname{IrNH} \mathrm{H}_{2}: \mathrm{C}^{\wedge} \mathrm{N}-2-\mathrm{pq}$ IrNH
IrNH

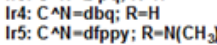

. Nagasawa et al. ${ }^{3}$ developed a NIR fluorescent probe, GPU-167, with longer Ex/Em wavelengths of $753 / 778 \mathrm{~nm}$, good hydrophobicity index, and aqueous solubility for in vivo optical imaging of tumor hypoxia using mouse xenograft models. GPU- $167^{3}$ is composed of tricarbocyanine dye as a NIR fluorophore and two 2-nitroimidazole moieties as exogenous hypoxia markers that become entrapped in hypoxic cells after reductive activation. After treatment with GPU167 , tumor cells contained significantly higher levels of fluorescence 
intensity at Ex/Em wavelength of $740 \mathrm{~nm} / 780 \mathrm{~nm}$ due to binding of GPU-167 to hypoxic cells in vitro. In vivo fluorescence imaging specifically detected GPU-167 in tumors after $24 \mathrm{~h}$ of administration and ex-vivo analysis revealed a strong correlation between fluorescence and hypoxia inducible factors (HIF)-1 active hypoxic regions using $71015 \mathrm{~nm}$ and $80010 \mathrm{~nm}$ as excitation and emission filter, respectively. ${ }^{32}$

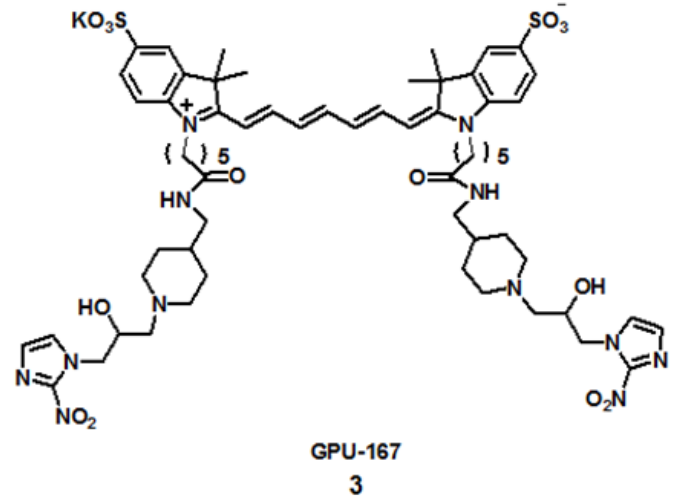

A "turn-on" NIRF probe GPU-327 (4a) has been used to evaluate both mild and severe hypoxia in various ocular ischemic diseased animal models. GPU-327 contains a NIR fluorophore as a PIET donor and a nitroaromatic moiety as a PIET modulator in which electron transfer from the excited fluorophore to the nitroaromatic moiety led to fluorescence quenching. On the other hand, under hypoxic conditions reductive metabolism cancels the PIET process, causing fluorescence "turn-on" due to the formation of hydroxyamino, nitroso, or aminobenzene moieties. GPU-328 (4b) which could be one of the metabolites of GPU-327 under hypoxic conditions was also synthesized and studied for its photophysical properties. GPU327 showed negligible fluorescence properties whereas GPU-328 exhibited near IR fluorescence at $788 \mathrm{~nm}(\Phi \mathrm{fl}=0.099)$. GPU-327 was injected in various hypoxic diseased mouse and rabbit models followed by non-invasive NIRF imaging in the ocular fundus using commercially available cameras. The fluorescence of GPU-327 increased under mild hypoxic conditions in vitro with excellent signalto-noise ratio without washing out during in vivo experiments, thereby enabling the imaging of deeper ischemia. GPU-327 could hence detect hypoxic areas successfully both in vivo and in vitro without causing neurotoxicity. ${ }^{33}$

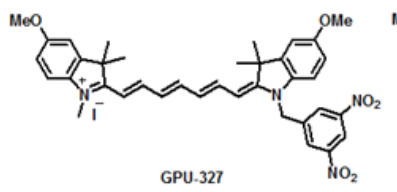

4 a

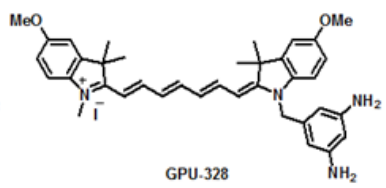

$4 \mathrm{~b}$
Here are the few examples of probes that monitor hypoxia by estimating target analytes, which are expressed in abnormal concentrations as a result of the reductive stress caused by hypoxic environment. The azide-based dye has been used as a fluorogenic probe to image hypoxia in human cancer cell lines. The non-fluorescent probe (5a) containing an azide group conjugated to an extended $\pi$-system is reduced by cytochrome P450 enzymes in an oxygen-dependent manner to give the corresponding fluorescent amine (5b). The reduction mechanism could be explained because of the coordination of azide to the heme center via a nitrenoid intermediate in hypoxia, whereas under normoxic conditions such binding is not observed as the iron center is blocked by oxygen. The fluorescence spectrum showed that the corresponding amine $(\mathrm{CH}-02 \mathrm{~F})$ was $\sim 600$ times more fluorescent than the azide-based probe $(\mathrm{CH}-02)$ with emission maxima at $625 \mathrm{~nm}$ upon excitation at $515 \mathrm{~nm}$. CH-02 could be used to determine cellular hypoxia by confocal microscopy and showed an increase in red fluorescence from normoxia to hypoxia. Fluorescenceassisted cell sorting (FACS) analysis revealed a 10 fold increase in fluorescence under hypoxic conditions when compared to normoxia. Furthermore, it was shown that $\mathrm{CH}-02$ could detect hypoxia in 3D tumor models because of its permeability across multiple cell layers. ${ }^{34}$

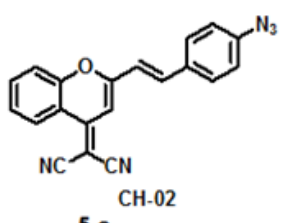

5 a

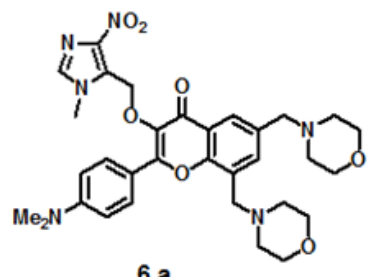

6 a
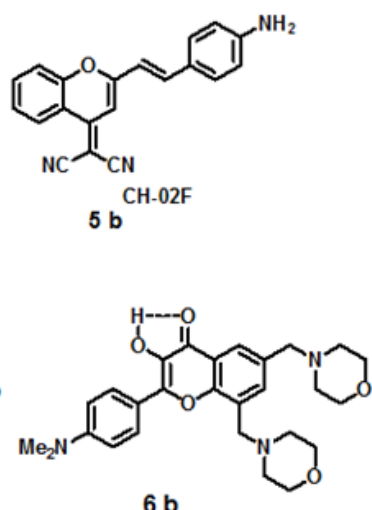

$6 \mathrm{~b}$
The "off-on" fluorescent probe (3-HF-NO, $6 a)$ was developed containing a 4-nitroimidazole moiety as a hypoxic trigger and two morpholine groups into the 3 -HF scaffold at the 6, 8-position for detection of nitroreductase (NTR), which is directly correlated with hypoxic status in solid tumors. 3-HF- $\mathrm{NO}_{2}$ exhibited two absorption bands with absorption maxima at $314 \mathrm{~nm}$ and $402 \mathrm{~nm}$. The nonfluorescent property and low background signal of 3-HF- $\mathrm{NO}_{2}$ could be attributed to PIET and blocking of ESIPT by 4-nitroimidazole. However, the presence of nitroreductase (NTR) and NADH in the probe $3-\mathrm{HF}-\mathrm{NO}_{2}$ buffer solution produced strong green fluorescence with emission maximum at $560 \mathrm{~nm}$ upon excitation at $412 \mathrm{~nm}$ exhibiting a detection limit of $63 \mathrm{ng} / \mathrm{mL}$ for NTR. Under hypoxic conditions, reduction of the 4-nitroimidazole moiety via a series of one-electron reduction processes to form an unstable 4-aminomidazol derivative followed by rearrangement-elimination leading to the release of the 3 -HF free dye (6b) formed the mechanistic basis for selective NTR detection. On the other hand, under normoxic conditions, the reduction of 3-HF- $\mathrm{NO}_{2}$ was suppressed by molecular oxygen. This probe was also successfully applied for imaging the hypoxic status of tumor cells (e.g., HeLa cells) with no cytotoxicity. ${ }^{35} \mathrm{~A}$ "turn-on" fluorescent probe semi-CyHP (7a) containing $p$-nitrophenyl group tethered to the indole moiety could detect NTR with strong fluorescence emission corresponding to semi-CyHF (7b). The fluorescence-quenching by the $p$-nitrophenyl group acts as a receptor for NTR and is converted to $p$-aminophenyl under hypoxic conditions. The probes semi-CyHP and semi-CyHF showed absorption maximum at $385 \mathrm{~nm}$ and 490 $\mathrm{nm}$, repectively. The fluorescence spectrum of semi-CyHF showed an emission peak at $556 \mathrm{~nm}$ when compared to weakly fluorescent semi-CyHP. The incubation of semi-CyHP with NTR in the presence of NADH led to 85-fold enhancement in fluorescence intensity at 556 $\mathrm{nm}$ in buffered aqueous solution on excitation at $490 \mathrm{~nm}$. The probe semi-CyHP showed no fluorescence under normoxic conditions in A549 cells whereas drastic fluorescent spots appeared within cells under hypoxic conditions with minimal endogenous interference during investigation of hypoxic tumor cell imaging. ${ }^{36}$ 


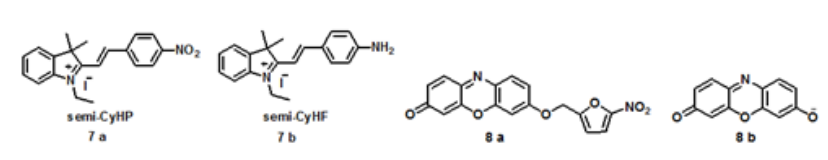

The nonfluorescent probe $8 \mathrm{a}$ reacts with NTR in the presence of $\mathrm{NADH}$ with the release of resorufin (8b) leading to pink and strong red fluorescence. Colorless probe $8 \mathrm{a}$ exhibited a weak absorption in the long wavelength region but the addition of NTR produced a strong absorption band at $550 \mathrm{~nm}$ with a color change to pink. In the fluorescence spectrum, the latent probe itself had negligible emission with low quantum yield of 0.04 due to alkylation of the 7-hydroxy group of resorufin which on reaction with NTR led to 100 -fold fluorescence enhancement at $585 \mathrm{~nm}$ with change in fluorescent color in aqueous buffered solution. Therefore, the probe could detect NTR selectively with a detection limit of $0.27 \mathrm{ng} / \mathrm{mL}$ and could be used for imaging the hypoxic status of tumor cells as it could discern HeLa and A549 cells under normoxic and hypoxic conditions successfully. ${ }^{37} \mathrm{HHC}$ (9a), an intramolecular charge transfer (ICT) fluorophore which is a hybrid of a hemicyanine dye and 2-(2-hydroxyphenyl)-benzothiazole (HBT) has also been synthesized. In this system, the hemicyanine dye exhibited long-wavelength absorption and emission due to ICT while HBT displayed ESIPT (the excited state intramolecular proton transfer) type absorption and emission due to the electron donating hydroxyl group. On excitation at $530 \mathrm{~nm}$, fluorescence spectrum of HHC showed an emission band at $450 \mathrm{~nm}$ due to ESIPT of HBT and at $616 \mathrm{~nm}$ due to ICT pushpull electron system. To modulate the ICT and ESIPT, p-nitrobenzyl group was introduced to the hydroxyl group of HHC resulting in a fluorescence probe HHCP (9b) for selective and sensitive detection and intracellular imaging of nitroreductase. The design of the probe is based on the photochemical property of nitroreductase, a flavincontaining enzyme, which can reduce nitroaromatics under hypoxic conditions using NADH as an electron donor. The non-fluorescent HHCP showed enhanced fluorescence at $580 \mathrm{~nm}$ with increased NTR concentration in the presence of NADH with a detection limit of 4.15 ng per $\mathrm{mL}$ under physiological conditions. The potential of HHCP for hypoxic tumor cell imaging was demonstrated using HeLa cells incubated with HHCP by enhancement of the red fluorescence due to emission from HHC under hypoxic conditions. ${ }^{38}$
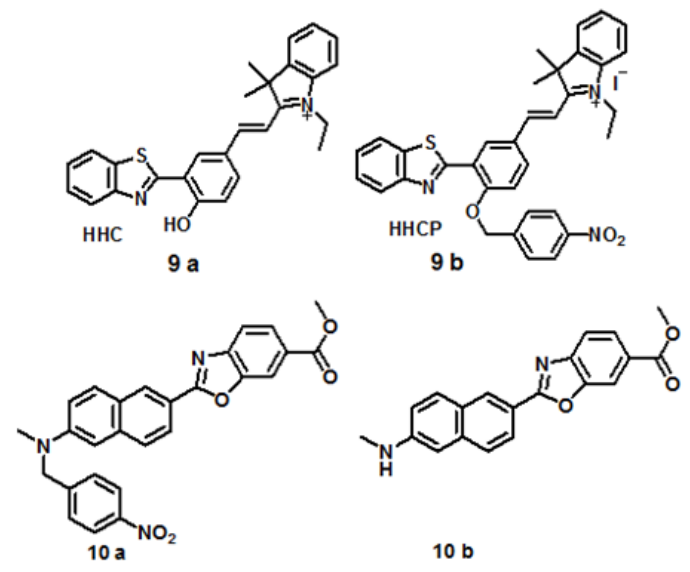

Two-photon fluorophores provided better three-dimensional spatial localization, higher imaging resolution, increased penetration depth and observation time along with decreased autofluorescence, and photobleaching over one-photon fluorophores in fluorescence studies for biological applications. ${ }^{39}$ In a key example, a two-photon
(TP) excited fluorescence probe 10a containing $p$-nitrobenzyl group could detect $20 \mathrm{ng} / \mathrm{mL}$ NTR with 70 -fold fluorescence enhancement at $494 \mathrm{~nm}$ in buffered aqueous solutions on excitation at $398 \mathrm{~nm}$. The reduction of probe $10 \mathrm{a}$ by nitroreductase and NADH was followed by rearrangement-elimination which released the free compound $10 \mathrm{~b}$ with green fluorescence emission. In addition, the probe was also used for one-photon and two-photon excited fluorescent bioimaging of hypoxic status in tumor cells and two-photon excited deep imaging of rat liver tumor tissue slices, with an imaging depth of $70-160 \mu \mathrm{m}$ in the green channel with $\lambda \mathrm{em} \sim 470-530 \mathrm{~nm} .{ }^{40}$ A non-fluorescent probe, NBP (11a), with an absorption peak at $525 \mathrm{~nm}$ could detect $180 \mathrm{ng} / \mathrm{mL}$ nitroreductase accompanied by fluorescence enhancement at $658 \mathrm{~nm}$ on excitation at $580 \mathrm{~nm}$ due to release of the Nile Blue fluorophores, NBF (11b). Further, fluorescent imaging of A549 cells also showed the ability of NIR probe NBP to detect tumor hypoxia with minimal endogenous interference. ${ }^{41}$

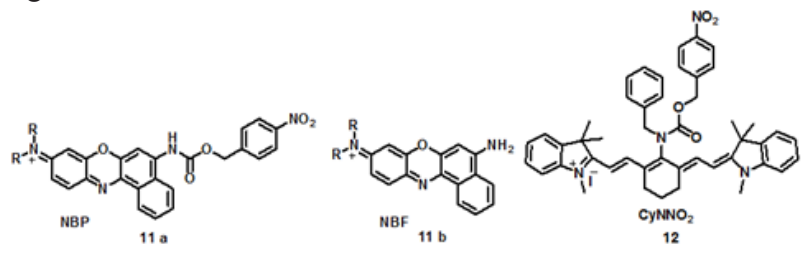

A near-infrared ratiometric fluorescent probe $\mathrm{CyNNO}_{2}$ (12), containing $p$-nitrobenzyl moiety as a reactive group, selectively estimated nitroreductase with a $0.0058 \mathrm{ng} / \mathrm{mL}$ limit of detection. The absorption maxima of $\mathrm{CyNNO}_{2}$ exhibited gradual decrease in intensity at $782 \mathrm{~nm}$ on incubation with nitroreductase accompanied by an increase in absorbance intensity at $645 \mathrm{~nm}$ with an isosbestic point at $689 \mathrm{~nm}$. The emission maxima on excitation in fluorescence spectrum of $\mathrm{CyNNO}_{2}$ at $805 \mathrm{~nm}$ (at $\lambda_{\text {max }} 689 \mathrm{~nm}$ ) showed enhancement on addition of nitroreductase and NADPH because of the interaction between $\mathrm{CyNNO}_{2}$ and nitroreductase. However, with reaction progression, the fluorescence intensity at $805 \mathrm{~nm}$ decreased along with an appearance of a new band at $747 \mathrm{~nm}$. These observations are attributed to the mechanism in which the carbamate group was cleaved, leading to the release of free benzylamine-substituted tricarbocyanine. The detection is based on the selective reduction of the nitro group to a hydroxylamine or amino group by nitroreductase in the presence of NADPH as an electron donor. It was demonstrated by confocal fluorescence imaging that $\mathrm{CyNNO}_{2}$ could detect intracellular hypoxia in A549 tumor cells incubated with $\mathrm{CyNNO}_{2}$ by imaging nitroreductase by dual emission ratiometry, which showed emission channels of 700-770 nm and 780-800 nm on excitation at $635 \mathrm{~nm} \cdot{ }^{42} \mathrm{~A}$ "turn-on fluorescent" NIR probe AZO-DCM (13a) was designed for selective and sensitive in vivo detection of cytochrome $\mathrm{P} 450$ reductase which is overexpressed in hypoxic tumor cells. The probe is based on the photo-stability of fluorophore dicyanomethylene-4H-pyran dye with an azo bond as probe quencher and hypoxic trigger. The reaction between probe and cytochrome $\mathrm{P} 450$ reductase led to the cleavage and reduction of azo bond to amine with the release of the DCM dye (13b) accompanied by 150 -fold increase in fluorescence within 4 minutes. The UV absorption spectrum of the probe AZO-DCM and fluorophore DCM showed $\lambda_{\max }$ at $520 \mathrm{~nm}$ and $500 \mathrm{~nm}$ in PBS buffer, respectively. The fluorescence spectrum of AZO-DCM did not show any fluorescent signal due to depletion of absorbed energy as a result of the vibration of the azo bond; whereas DCM showed a strong fluorescence at $650 \mathrm{~nm}$. It was also shown by hypoxic cell imaging (A549 cells) that AZO-DCM is highly selective to $\mathrm{pO}_{2}$ with 152 -fold increase in mean fluorescence intensity under hypoxic condition. Therefore, it could be inferred that the azo bond is an excellent potential receptor for hypoxia detection. ${ }^{43}$ 

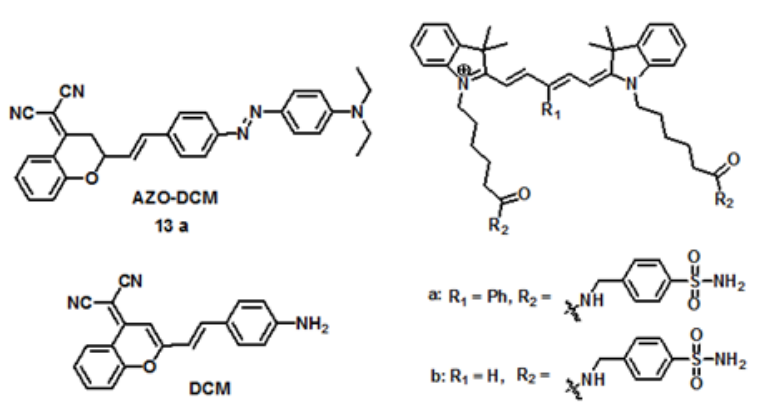

$13 \mathrm{~b}$

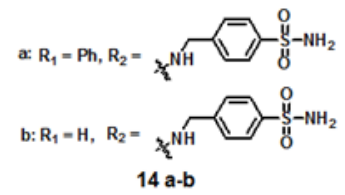

Tan and co-workers strategically designed and created analyte responsive NIR fluorogenic probes containing a small molecule ligand conjugated to a $\gamma$-phenyl-substituted Cy5 fluorophore for selective detection of proteins through a non-enzymatic process. The self-assembled fluorescence quenched J-type aggregate of probes in the presence of the target protein emitted bright fluorescence in the near-IR region. Probe 14a showed fluorescence enhancement by 48fold at $648 \mathrm{~nm}$ in the presence of hCAII protein with a $17 \mathrm{nM}$ limit of detection which was credited to the self-assembly/disassembly of Cy5 fluorophore dye as a result of the recognition-induced disassembly of the probe aggregate. Whereas probe $14 \mathrm{~b}$ showed only 2 -fold fluorescence enhancement in the presence of hCAII due to the absence of $\gamma$-substituted phenyl group at the pentamethinium bridge. The significant role of $\gamma$-phenyl-substitution in Cy5 fluorophore was supported by the observation that the classical Cy5 dye without $\gamma$-phenyl-substitution caused no fluorescence increase. Probe 14a was used for no-wash imaging of tumor cells (HeLa cells) for the detection of hypoxia-induced cancer-specific biomarker, transmembrane-type carbonic anhydrase IX. ${ }^{44}$

A series of NIR fluorescent sulfonamides ${ }^{15-18}$ as hCAIX inhibitors for non-invasive in vivo detection and quantification of hypoxia induced hCAIX expression have been reported. The compounds caused inhibition of transmembrane hCAIX with different potencies ranging from $7.5-116 \mathrm{nM}$ and up to a 50 -fold selectivity over the cytosolic form hCAII. In addition to this, some of the compounds showed inhibition selectivity for transmembrane hCAXII and XIV. Fluorescence microscopy results showed the upregulation of hCAIX preferentially under hypoxic conditions in HeLa cells cultured under both normoxic and hypoxic environments. In the live animal studies these compounds were able to quantitatively image hCAIX upregulation. A pilot in vivo study of HT-29 tumor bearing mice showed significant accumulation of a fluorescent acetazolamide derivative in tumor tissue when compared to other tissues and $10 \%$ of injected dose was non-invasively quantified in tumors by fluorescence molecular tomography (FMT)..$^{45}$
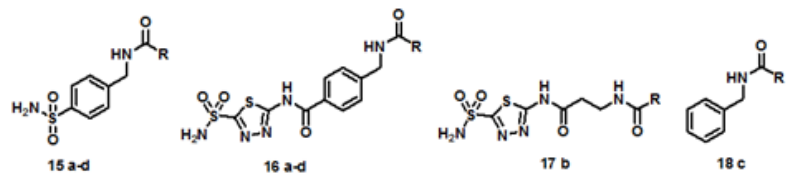

A group of NIRF (near-infrared fluorescence) heptamethine carbocyanine dyes and derivative NIRF dye-drug conjugates have been synthesized for detection of primary and metastatic brain tumors in mice which was mediated by tumor hypoxia and organic aniontransporting polypeptide genes (OATPs). It was demonstrated by both in vivo and ex vivo NIRF imaging that IR-783 (19a) was taken up specifically by the tumor bearing brains in mice, which also showed up to a 82.6-fold higher NIRF signal intensity in the brain as compared to that in other selected organs. NIRF dye can be taken up by brain tumors after effectively penetrating the BBB/BTB (bloodbrain barrier/ blood-tumor barrier). Heptamethine carbocyanine dyes having charged side-chain terminal groups and a chlorine atom at the central meso position of the cyclohexyl ring and a negatively charged IR-783 or MHI-148 possessing a sulfate/carboxyl group are able to cross BBB. Also, these dyes in conjugation to chemotherapeutic agents such as gemcitabine (19b), significantly restricted the growth of both intracranial glioma xenografts and prostate tumor brain metastases and prolonged survival in mice. ${ }^{46} \mathrm{~A}$ NIRF imaging agent, MHI-148 dye, ${ }^{20}$ was used for detection of hypoxia in cancer cells through monitoring hypoxia inducible factor as a key mediator for in vitro and in vivo experiments. The cancer cells used genitourinary cancers as representative model systems and tumor xenografts exhibited hypoxia-dependent MHI-148 dye uptake leading to an increase of NIRF signals. This was directly mediated by hypoxiainducible factor $1 \alpha(\mathrm{HIF} 1 \alpha)$ and a group of hypoxia-inducible organic anion-transporting polypeptides (OATPs) as shown by microarray analysis and dye uptake assay in situ in perfused clinical tumor samples. ${ }^{47}$
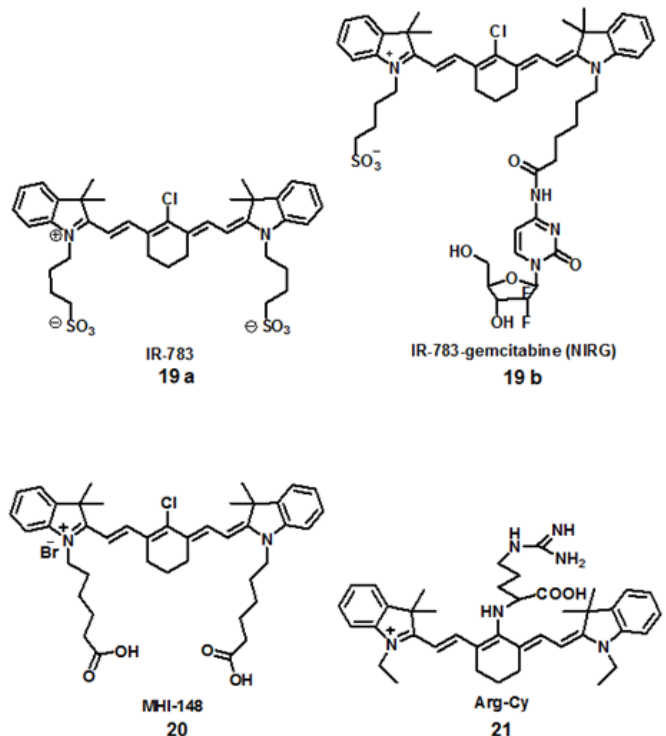

A near infrared fluorescent probe Arg-Cy] was synthesized by conjugating $\mathrm{L}$-arginine to tricarbochlorocyanine dye (Cy.7.Cl) exhibiting excitation and emission spectra at $680 \mathrm{~nm}$ and $749 \mathrm{~nm}$, respectively. The probe (Arg-Cy) monitored the metabolic products of the reducing agent ascorbic acid (AA) in living cells based on the reaction of the guanidine group in Arg-Cy with the adjacent diketone involved in the metabolites of AA. The probe could successfully visualize L-xylosone levels in living cells under normoxic conditions accompanied by higher $\mathrm{H}_{2} \mathrm{O}_{2}$ levels. However, in the hypoxic tumor microenvironment, the L-xylosone levels monitored during the process of HepG2 cell death induced by pharmacological doses of AA, showed no change in fluorescence and $\mathrm{H}_{2} \mathrm{O}_{2}$ levels. The AA metabolized into L-xylosone only under normoxic condition due to oxidative stress but not under hypoxic conditions. A related metabolite used for detection of hypoxic environment in biological systems, DHA (dehydroascorbic acid), was selected instead of AA, as it is known that AA enters cells in the form of DHA in most cancer cells. Fluorescence studies in aqueous buffered solutions revealed that on 
addition of DHA to disrupted (lysed) cell liquids already containing Arg-Cy there was an increase in fluorescence intensity at $749 \mathrm{~nm}$, indicating the response of Arg-Cy towards metabolites of DHA under hypoxic conditions. Similarly, the Hep G2 cells already loaded with probe showed bright red fluorescence with DHA. ${ }^{48}$

An injectable PTD-ODD-luciferase-NIR fluorescent dye (POL-N) has been developed, which generated NIR-BRET (bioluminescence resonance energy transfer) signals in a luciferase substrate-dependent manner for detection of ubiquitin-regulated hypoxia-inducible factor (HIF) activity in vivo. Because polyubiquitin tagging of proteins facilitates selective degradation into small peptides by the proteasome, it requires ubiquitin-activating enzyme (E1), ubiquitin-conjugating enzyme (E2), and ubiquitin-ligase (E1). ${ }^{49}$ As the luciferase is fused to the E3 ligase-recognition domain of the HIF- $1 \alpha$, POL-N is intact only in the HIF $\alpha$-overexpressing cells. HIF-active cells generated signals as a result of the intramolecular BRET between luciferase and a nearinfrared (NIR) fluorescent dye, where the dye was located at the C-terminal end of the probe. POL was labeled with a NIR fluorescent dye, AlexaFluor 680 (AF) which exhibited fluorescence at $702 \mathrm{~nm}$ on excitation at $684 \mathrm{~nm}$ resulting in a large overlap between the donor (luciferase) bioluminescence and the acceptor (AF) absorption spectra. The BRET signal generated at $\lambda_{\max } 702 \mathrm{~nm}$, generated from POL-AF was highly efficient in penetrating the biological tissues as compared to the original bioluminescence at $\lambda_{\max } 547 \mathrm{~nm}$ from POL. The correlation between BRET signal intensity and HIF- $1 \alpha$ protein expression was examined in LM9/HRE-Fluc and SUIT-2/HRE-Fluc cells which showed that the BRET signal intensity quickly decreased in normoxic cells, where HIF-1 $\alpha$ was not detected, but increased in hypoxic cells, in which HIF-1 $\alpha$ accumulated. The intratumoral activity could be detected in a selective and fast manner as off-target signals of the NIR-BRET were very low. The probe successfully detected hypoxic liver metastasis within one hour of systemic injection of probe, which is difficult to detect by injectable imaging probes due to strong off-target signals. ${ }^{50}$

\section{Macromolecular and chelator based probes}

Macromolecular near-infrared imaging agents, two-photon luminescent probes, as well as luminescent transition metal complexes, and cationic conjugated polymers have been reported to overcome the anomalies of small/dye based molecular probes. They provide advantages of tunable photo-physical properties such as emission at longer wavelengths to minimize interferences arising from within biological samples (i.e., auto-fluorescence), imaging cycling hypoxia in vivo, and high luminescent quantum yields. As detailed in this section, various macromolecular and chelator based probes detect the hypoxic environment either by directly monitoring oxygen levels or by indirectly measuring hypoxia inducible factors such as nitroreductase, carbonic anhydrase or $\mathrm{pH}$ value.

Very few probes are known in literature for direct identification of hypoxic cells. Most of the methods reported visualize hypoxia in tumor tissues using radioactive isotopes,${ }^{51}$ but there are reports that have also reflected the interest of having a noninvasive imaging technology available to detect hypoxia in retinal tissues. ${ }^{52}$ Traditional technologies used for studying retinal hypoxia include pimonidazole mediated immunohistochemistry which have been limited to $e x$ vivo methods of evaluation. In such model systems of retinopathy, hypoxia has shown to lead to reductive stress resulting in increased expression of reductases at the lesion. ${ }^{53}$ Based on the feature of having reductase induced cleavage of azo-bonds, an optical imaging probe known as HYPOX-3 (22) has been developed and contains a hypoxia sensitive cleavable azo-bond which links a NIR dye to a fluorescence quencher. The fluorescence intensity at $704 \mathrm{~nm}$ of the HYPOX-3 was found to increase by 6.6 fold upon excitation at 670 $\mathrm{nm}$ after undergoing 4 hours of a hypoxic environment. The probe could detect oxygen concentrations down to $0.1 \%$ with significant fluorescence enhancement. Importantly, the HYPOX-3 showed no acute toxicity, and it was found to be capable of detecting hypoxia by undergoing cleavage in hypoxic retinal cell culture and mouse models with a high signal-to-noise ratio; whereas HYPOX-3 fluorescence was undetectable in hypoxic cells and tissues under normoxia. These results are a promising move towards examinations with NIR probes for imaging hypoxia in preclinical disease models and in the future for patients. ${ }^{54}$

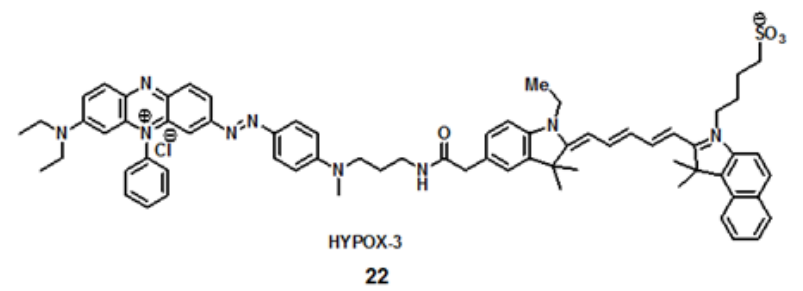

In order to improve the pharmacokinetic parameters of the previous HYPOX-3 probe, ${ }^{54,55}$ HYPOX-4 was designed to allow enhanced diffusion into regions with limited vasculature for discriminating normoxia and hypoxia in vivo. Using the same means of enhancing the fluorescence via hypoxia induced reductases, the response of HYPOX-4 towards hypoxia in vivo and ex vivo was tested in a mouse model of oxygen-induced retinopathy with no apparent effect on cell proliferation and no acute toxicity. ${ }^{56}$ It has been reported in literature that cycling hypoxia (recurrent hypoxia and reoxygenation) induces the up-regulation of HIF-1 transcription factor in tumor cells, thereby lowering the effectiveness of cancer treatments including radiotherapy or chemotherapy. ${ }^{57}$ For selective and dynamic monitoring of cycling hypoxia in live cells, three reversible ruthenium (II) anthraquinone complexes (RuL1-3) (23) have been developed as two-photon luminescent probes. The purpose of the design was to combine a luminescent ruthenium (II) complex, as a sensitizer, with a redox-active anthraquinone moiety, as a quencher. Due to their ability to accept electrons, quinones can serve as capable quenchers of fluorescence. The redox switch of the quinone/hydroquinone pair in the probe was afforded by reductases under hypoxic condition for the formation of the hydro-anthraquinone that was capable of two-photon luminescence. A corresponding imaging technique was effectively applied by implementing these probes to visualize hypoxia in tumor spheroids and to detect repeated cycles of hypoxia/reoxygenation in living zebrafish. Under hypoxic conditions, the reduction of nonluminescent RuL1-3 by reductases produced an 80 -fold luminescent enhancement at $615 \mathrm{~nm}$ upon excitation at $458 \mathrm{~nm}$ with a luminescent quantum yield $(\Phi)$ of $0.40-0.92 \%$ when compared to normoxic conditions. A critical part of the process was the ability of the probe to function in a fully reversible and repeatable manner in order to afford an "off-on" luminescent switching process when tested in buffered solution containing rat liver microsomes. ${ }^{58}$
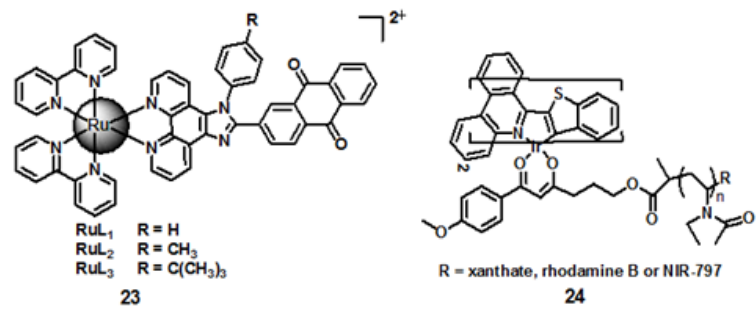
An example of an oxygen sensitive macromolecular probe has been developed which makes use of emission in the near-infrared (24) for in vivo monitoring of the hypoxic tumor environment of various cancer models. This poly(N-vinylpyrrolidone) (PVP)-conjugated iridium (III) complex probe (Ir-PVP) was found to be able to generate a significantly higher luminescent intensity as compared to commercial oxygen probes. Probe Ir-PVP (24) utilizes the Ir(III) complex along with its highly conjugated ligand to extend the luminescence into the near IR region. The use of molecular oxygen itself quenches the luminescence; hence, this method is highly specific for hypoxia induced emission. By linking the PVP to rhodamine B as an internal standard, the probe was able to provide a means for ratiometric measurements, with Ir-PVP-RhB measured hypoxia in SH-SY5Y cells showing red emission due to increased emission intensity of the Ir moiety at $670-750 \mathrm{~nm}$. In vivo imaging of tumor hypoxia was highly effective and because of the reduced clearance of PVP the probe measurement could be taken over 120 hours. ${ }^{59}$

Recently, a fluorescent cationic conjugated polymer (PBFBT-NP, 25 ) probe containing $p$-nitrophenyl groups in the side chain has been reported for detection of nitroreductase (NTR), which is indicative of hypoxia. PBFBT-NP showed two absorption peaks at 350 and 430 $\mathrm{nm}$, respectively. The fluorescence of PBFBT-NP is quenched due to photo-induced electron transfer (PIET) which in the presence of nitroreductase leads to a 110 -fold fluorescent enhancement at $530 \mathrm{~nm}$. The enhancement occurs with excitation at $430 \mathrm{~nm}$ as a result of the specific conversion of the $p$-nitrophenyl group to $\mathrm{p}$-aminophenyl by the nitroreductase; thereby leading to inhibition of the (PIET). The significant increase in the emission intensity of the probe afforded as low as $2.9 \mathrm{ng} / \mathrm{mL}$ of nitroreductase to be detected in vitro and furthermore it was shown that the hypoxia level in tumor cells could be visualized by fluorescence microscopy with a very strong green fluorescence in the nucleus for cells exposed to anaerobic conditions as compared to a weak green signal under normoxic conditions. ${ }^{60}$

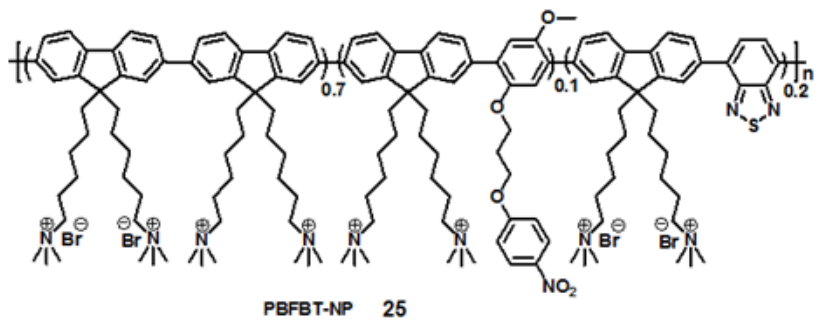

Near-infrared fluorescence imaging probes could also be utilized to monitor hyperbaric oxygen therapy (HBOT) which is used in the treatment of radiation-induced tissue injury. Because tumor hypoxia has been found to induce the upregulation of carbonic anhydrase IX (CA IX), near-IR hypoxia probes including Hypoxisense680 have been designed based on fluorescently labeled derivatives of the CA IX inhibitor acetazolamide. Studies with these probes have revealed that HBOT treatment caused an accelerated growth of non-irradiated tumors and enhanced hypoxia. During the last stages of HBOT sessions, mice were intravenously injected with the fluorescent carbonic anhydrase IX targeted imaging agent Hypoxisense680 for fluorescence molecular tomography (FMT) imaging. Such use of this in vivo near infrared fluorescence optical imaging technique allowed for the influence of HBOT on the hypoxic state of squamous cell carcinoma to be assessed and this probe has become a valuable tool for in vivo imaging and drug screening in rodent models. ${ }^{61,62}$

\section{Nanoparticle based probes}

Nanoscale imaging probes such as quantum dots, magnetic nanoparticles, and carbon nanostructures have attracted attention for medical application because of their robust and customizable physical properties. In one class of nanomaterial, upconversion nanoparticle (UCNP)-based nanosensors have been explored for oxygen sensing either as thin films or UCNP core/dense silica nanoparticles. ${ }^{63}$ Other fluorescent nanoprobes include water dispersible nanoparticles comprised of $\pi$-conjugated polymers (CPs), which offer an inherent advantage of low-bandgap as compared to typical CPs. ${ }^{64} \mathrm{In}$ this section we will discuss these and other such inorganic nanoparticle based probes that can provide magnificent opportunities for in vivo imaging.

Due to the enhanced signal that quantum dots (QDs) can provide, as well as their inherent capability to not undergo photo-bleaching as compared to traditional organic fluorophores, QDs have been applied in recent years to biomedical imaging of hypoxia in deep tissue. In addition, in vitro assessment of oxygen levels have also made use of QDs, specifically a NIR emissive AgInS2/ZnS QD coupled to a perylene dye ${ }^{26}$ has shown to serve as an effective ratiometric probe. The response of the probe towards varying oxygen concentrations in hypoxia was studied using enzymatic oxygen scavenging in water using glucose, glucose oxidase, catalase, and ATP to deoxygenate samples. The probe showed more than a $100 \%$ increase in ratiometric signal upon deoxygenation from 6.8 to $0.01 \mathrm{ppm}$. The ratiometric response of the probe to oxygen concentration was accompanied by emission of the perylene dye at $472 \mathrm{~nm}\left(\lambda_{\max } 427 \mathrm{~nm}\right)$ and that of the QD at $775 \mathrm{~nm}\left(\lambda_{\max } 365 \mathrm{~nm}\right){ }^{65}$

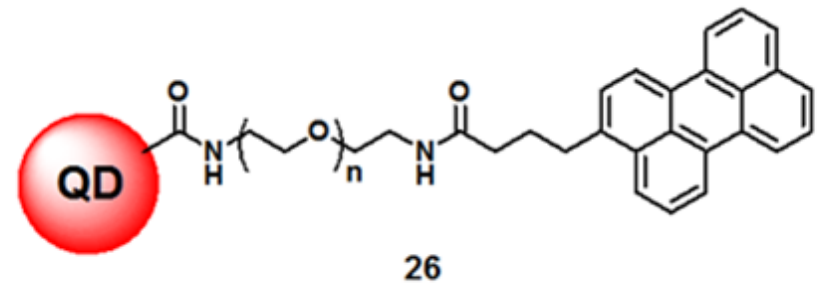

An oxygen nanoprobe made up of mesoporous silica coated lanthanide-doped upconversion nanoparticles (UCNPs) covalently attached by oxygen-sensitive Ir (III) complex (Figure 1) in the outer silica shell (denoted as UCNPs@mSiO - -Ir) could image intracellular hypoxia by using time-resolved luminescent imaging technology and near-infrared light excitation under both downconversion and upconversion channels. Upconversion luminescence (UCL) spectra of nanoprobe showed emission from complex Ir at $600 \mathrm{~nm}$ under NIR excitation of $980 \mathrm{~nm}$ due to energy transfer from UCNPs to complex Ir. The emission intensity between $560-630 \mathrm{~nm}$ decreased significantly with increase of oxygen concentration whereas the UCL of UCNPs is insensitive to oxygen concentration. The nanoprobe under the downconversion channel showed decrease of phosphorescence intensity at $600 \mathrm{~nm}$ with increasing oxygen concentration upon excitation at $450 \mathrm{~nm}$. The emission lifetimes of the nanoprobe also reduced from $4031 \mathrm{~ns}$ to $836 \mathrm{~ns}$ at $\mathrm{O}_{2}$ concentrations of $0 \%$ to $21 \%$, respectively. In vitro tests confirmed that this probe was capable of intracellular hypoxia imaging in HeLa cells. ${ }^{66}$

In an upconversion LRET (luminescence resonance energy transfer) nanosensor, $[\mathrm{Ru}(\mathrm{Dpp}) 3] 2+\mathrm{Cl}_{2}$ acted as an oxygen indicator and upconversion nanoparticles (UCNPs) acted as energy donors. Specifically, the UCNPs provided the excitation light for $[\mathrm{Ru}(\mathrm{Dpp}) 3] 2+\mathrm{Cl}_{2}$ by the upconversion process under $980 \mathrm{~nm}$ excitation (Figure 2). The reported nanosensor detected hypoxic conditions reversibly by displaying luminescence, which was unaffected by acidic and reducing microenvironments in the cancer cells with enhanced sensitivity as compared to free $[\mathrm{Ru}(\mathrm{Dpp}) 3] 2+\mathrm{Cl}_{2}$. UCNP-based nanosensors enabled ratiometric sensing of hypoxia at UCL (upconversion luminescence) ratio (UCL613nm/ UCL477nm) 
as the red emission band at $613 \mathrm{~nm}$ was greatly quenched at higher oxygen levels when compared to the emission band at $477 \mathrm{~nm}$. Confocal imaging of living U87MG cells as well as zebrafish embryos also showed that the signal was reversible upon repeated exposure to hypoxia and subsequent normoxia conditions showed a clear luminescent quenching and recovery, respectively. ${ }^{67}$

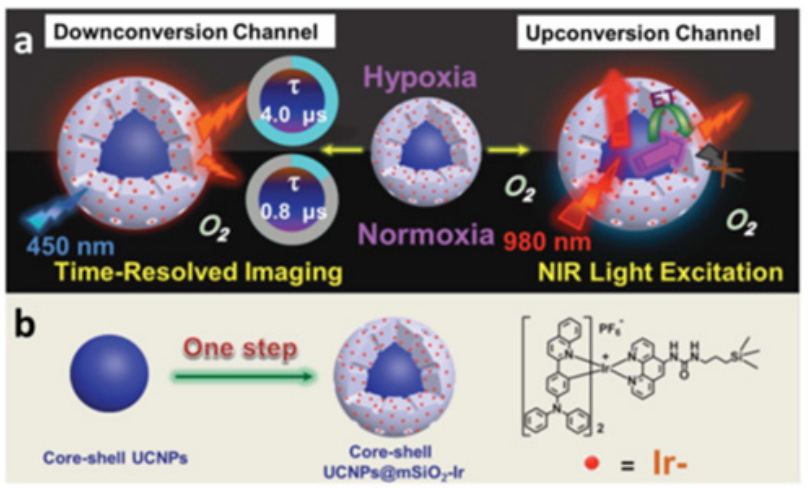

Figure I a) Mechanism and b) synthesis of core-shell UCNPs@mSiO2 Ir. (Figure reproduced from reference; ${ }^{66}$ use permitted under the Creative Commons Attribution License CC BY 4.0.).

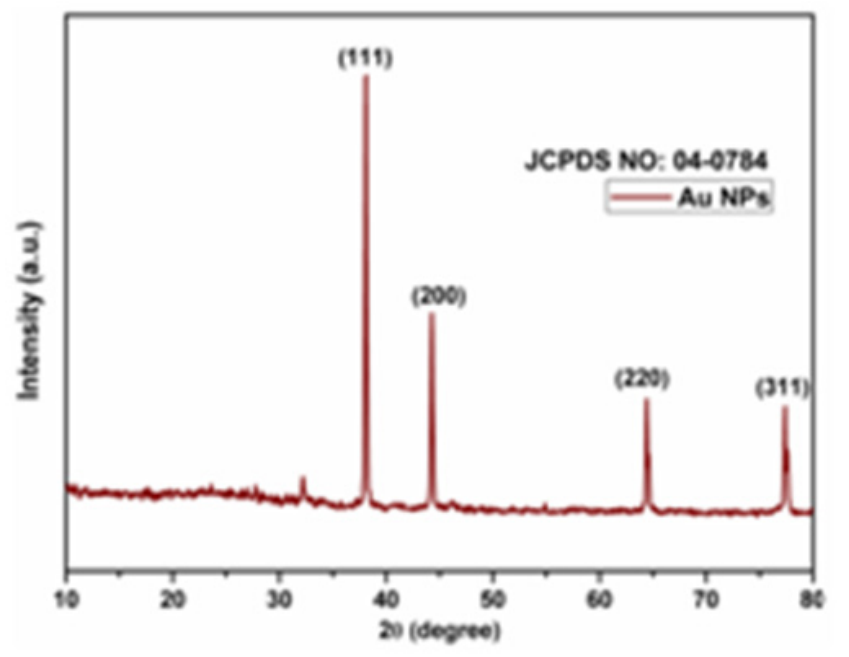

Figure 2 Overview of the use of UCNP for LRET based detection of oxygen. (Figure reprinted with permission from reference. ${ }^{67}$

In other recent implementations of nanoparticle-based probes, low band gap conjugated polymers such as diketopyrrolopyrrole have been used for their excellent stability and high quantum yield. In one such example for hypoxia detection, researchers have taken advantage of diketopyrrolopyrrole with polystyrene as the organic photonic core and encapsulated this in the pluronic F-68. Additional blending of the cores with the additive platinum benzoporphyrin (PtBzPor) facilitated an oxygen responsive components as the near-infrared phosphorescence is altered in the presence of oxygen. As a result, a ratiometric signal from the two luminescent components could afford two-emissions wherein the oxygen content could be observed by the "turn-off" of the PtBzPor signal in relation to the internal standard fluorescence of the diketopyrrolopyrrole. This functional scheme benefited by having the excitation of both luminescent components in the blend at $610 \mathrm{~nm}$ while the emissions were distinct. The phosphorescence at $760 \mathrm{~nm}$ of the oxygen sensitive PtBzPor could be quenched upon diffusion of oxygen molecules while the diketopyrrolopyrrole luminescence at $680 \mathrm{~nm}$ could be used for normalization of the signal. The spherical core of the nanoparticles was found to be highly $\pi$-electron rich due to PS (polystyrene) as well as the high electron density of the PFDPP (the diketopyrrolopyrrole with a semi-fluorinated side chain pluronic). Because of the particle's small size, approximately $20 \mathrm{~nm}$, they were capable of effective circulation as well as minimal uptake in the liver and kidney as seen after intravenous injection in mice. Noteworthy, this non-toxic nanoparticle blend was successfully used to detect pathological tissue deoxygenation at hypoxic sites in a ratiometric manner by interpreting the relative luminescence intensities. ${ }^{68}$

\section{Conclusion}

This review illustrates the design and sensing mechanism of various fluorescent and luminescent probes based on small molecules, dyes, inorganic nanoparticles, quantum dots, and macromolecules for detection of hypoxic conditions non-invasively; either by measuring oxygen levels or detecting targeted hypoxia inducible factors. It has been proven that near-infrared fluorescence (NIRF) imaging is successful for detection of hypoxia and its translation into human studies. NIRF using indocyanine green (ICG) has been reported to be implemented intraoperatively in sentinel lymph node mapping to potentially supplant blue-dye visualization of tumor-draining lymph nodes in a number of cancers. NIRF-ICG tomography has also been used along with mammography and ultrasonography to detect lesions and to differentiate between malignant and benign breast lesions based on ICG washout kinetics. NIRF imaging has advantages over traditional and frequently used tracer nuclear magnetic techniques in cancer treatments, as it lacks radioactivity and has high photon count rates for rapid and sensitive imaging, therefore can be used more effectively for intraoperative visualization. ${ }^{69}$ Such probes as these discussed above have shown noteworthy results which have already or are soon to find use in vivo. Among various probes, functional nanomaterials and quantum dots are found to be more attractive as compared to fluorescent dyes for hypoxia detection. Nanomaterials and quantum dots have particular benefits of broad excitation spectra, narrow defined tunable emission peaks, longer fluorescence lifetimes, resistance to photo-bleaching, and higher molar extinction coefficients. The resultant lowering of emission signal overlap offers brighter probes as compared to conventional fluorophores; but still careful evaluation of their potential toxicity is needed. ${ }^{70}$

Despite all the remarkable advances made in this rapidly emerging field of hypoxia detection using various formulations of fluorescent probes, there still persists considerable challenges for developing improved probes for in vivo imaging in a real clinical setting. The various factors such as auto-oxidizability, high photostability, biocompatibility, biodegradability, low interference from biological environments, and toxicity screening are required to be carefully monitored for developing in vivo bioimaging probes in clinical situations. Also, ratiometric fluorescence imaging and phosphorescence lifetime imaging should be explored along with traditional fluorescent/luminescent imaging for improved accuracy of output signals. Therefore, to achieve the optimum success in design and practical application of desired probes for hypoxia detection, it is imperative to speed up the interdisciplinary research collaborations amongst various fields such as chemistry, materials science, nanomedicine, and bioimaging in order to put continuous efforts for fruitful results in real world applications of cancer diagnosis through sophisticated investigative techniques.

\section{Acknowledgements}

The authors acknowledge the financial support from the National Heart, Lung and Blood Institute with the award number NIH T32 HL134613 LeNaiya Kydd. The content is solely the responsibility of the authors and does not necessarily represent the official views of the National Institutes of Health. 


\section{Conflicts of interest}

The authors declare no conflict of interest.

\section{References}

1. Till Acker, Helmut Acker Cellular oxygen sensing needs in CNS function: physiological and pathological implications. J Exp Biol. 2004;207:3171-3188.

2. Kizaka-Kondoh $\mathrm{S}$, Inoue $\mathrm{M}$, Harada $\mathrm{H}$ et al. Tumor hypoxia: a target for selective cancer therapy. Cancer Sci. 2003;94(12):1021-1028.

3. Huang Z, Shiva S, Kim-Shapiro DB et al. Enzymatic function of hemoglobin as a nitrite reductase that produces NO under allosteric control. J Clin Invest. 2005;115(8):2099-2107.

4. Murdoch $\mathrm{C}$, Muthana $\mathrm{M}$, Lewis $\mathrm{CE}$ Hypoxia regulates macrophage functions in inflammation. J Immunol. 2005;175(10):6257-6263.

5. Semenza GL Angiogenesis in ischemic and neoplastic disorders. Annu Rev Med. 2003;54:17-28.

6. Ferris FL, Davis MD, Aiello LM Treatment of diabetic retinopathy. Engl J Med. 2016;341(9):667-678.

7. Hayreh SS Pathogenesis of optic disc edema in raised intracranial pressure. Prog Retin Eye Res. 2011;30:359-394.

8. Kaanders JH, Wijffels KI, Marres HA et al. Pimonidazole binding and tumor vascularity predict for treatment outcome in head and neck cancer. Cancer Res. 2002;62(23):7066-7074.

9. Anderson CJ, Ferdani R Copper-64 radiopharmaceuticals for PET imaging of cancer: advances in preclinical and clinical research. Radiopharm. 2009;24:379-393.

10. Sakr TM, MA Motaleb, IT Ibrahim J Synthesis and biodistribution of $99 \mathrm{mTc}-\mathrm{PyDA}$ as a potential marker for tumor hypoxia imaging. Radioanal Nucl Chem. 2012;292:705-710.

11. Egeland TA, Gulliksrud K, Gaustad JV et al. Dynamic contrast-enhancedMRI of tumor hypoxia. Magn Reson Med. 2012;67(2):519-530.

12. Dai C, Liu X, Zhang HF, Puliafito CA, Jiao S Absolute retinal blood flow measurement with a dual-beam Doppler optical coherence tomography. Invest Ophthalmol Vis Sci. 2013;54(13):7998-8003.

13. Zhang S, Hosaka M, Yoshihara T et al. Phosphorescent light-emitting iridium complexes serve as a hypoxia-sensing probe for tumor imaging in living animals. Cancer Res. 2010;70(11):4490-4498.

14. Sun X, Niu G, Chan N, Shen B, Chen X Tumor hypoxia imaging. Mol Imaging Biol. 2011;13(3):399-410.

15. Liu JN, Bu W, Shi J Chemical Design and Synthesis of Functionalized Probes for Imaging and Treating Tumor Hypoxia. Chem Rev. 2017;117(9):6160-6224.

16. Chen $\mathrm{Y}, \mathrm{Hu} \mathrm{L}$ Design of anticancer prodrugs for reductive activation Med Res Rev. 2009;29(1):29-64.

17. Minchinton AI, Tannock IF Drug penetration in solid tumours. Nat Rev Cancer. 2006;6(8):583-592.

18. Wilson WR, Hay MP Targeting hypoxia in cancer therapy. Nat Rev Cancer. 2011;11(6):393-410.

19. Vaupel P, Harrison L Tumor hypoxia: causative factors, compensatory mechanisms, and cellular response. Oncologist. 2004;9(Suppl 5):4-9.

20. Agarwal S, Sane R, Oberoi R et al. Delivery of molecularly targeted therapy to malignant glioma, a disease of the whole brain. Expert Rev Mol Med. 2011;13:e17.

21. Behin A, Hoang-Xuan K, Carpentier AF et al. Primary brain tumours in adults. Lancet. 2003;361(9354):323-331.
22. Zhang B, Sun X, Mei H et al. LDLR-mediated peptide-22-conjugated nanoparticles for dual-targeting their apy of brain glioma. Biomaterials. 2013;34(36):9171-9182.

23. Kobayashi H, Ogawa M, Alford $R$ et al. New strategies for fluorescent probe design in medical diagnostic imaging. Chem Rev. 2010;110(5):2620-2640

24. Yuan L, Lin W, Zheng $\mathrm{K}$ et al. Far-red to near infrared analyteresponsive fluorescent probes based on organic fluorophore platforms for fluorescence imaging. Chem Soc Rev. 2013;42(2):622-661.

25. Woo Kyung Moon, Yuhui Lin, Terence O’Loughlin et al. Enhanced Tumor Detection Using a Folate Receptor-Targeted Near-Infrared Fluorochrome Conjugate Bioconjugate Chem. 2003;14:539-545.

26. Peterson JD, LaBranche TP, Vasquez $\mathrm{KO}$ et al. Optical tomographic imaging discriminates between disease-modifying anti-rheumatic drug (DMARD) and non-DMARD efficacy in collagen antibody-induced arthritis. Arthritis Research \& Therapy. 2010;12:105R.

27. Kossodo S, Pickarski M, SA Lin et al. Dual In vivo Quantification of Integrin-targeted and Protease-activated Agents in Cancer Using Fluorescence Molecular Tomography (FMT). Mol Imaging Biol. 2010;12:488-499.

28. Nahrendorf $\mathrm{M}$, Waterman $\mathrm{P}$, Thurber $\mathrm{G}$ et al. Hybrid in vivo FMTCT imaging of protease activity in atherosclerosis with customized nanosensors. Thromb Vasc Biol. 2009;29(10):1444-1451.

29. He X, Gao J, Gambhir SS, Cheng Z Near-infrared fluorescent nanoprobes for cancer molecular imaging: status and challenges Trends Mol Med 16(12):574-583.

30. Sevick-Muraca EM Translation of near-infrared fluorescence imaging technologies: emerging clinical applications. Annu Rev Med. 2012;63:217-231.

31. W Piao W, Tsuda S, Tanaka Y et al. Development of azo-based fluorescent probes to detect different levels of hypoxia. Angew Chem Int Ed Engl. 2013;52(49):13028-13032.

32. L Sun, G Li, X Chen et al. Azo-Based Iridium (III) Complexes as Multicolor Phosphorescent Probes to Detect Hypoxia in 3D Multicellular Tumor Spheroids. Scientific Reports. 2015;5:14837.

33. Okuda K, Okabe Y, Kadonosono $\mathrm{T}$ et al. 2-Nitroimidazoletricarbocyanine conjugate as a near-infrared fluorescent probe for in vivo imaging of tumor hypoxia. Bioconjugate Chem. 2012;23:324-329.

34. S Fukuda, K Okuda, G Kishino et al. In vivo retinal and choroidal hypoxia imaging using a novel activatable hypoxia-selective nearinfrared fluorescent probe. Graefes Arch Clin Exp Ophthalmol. 2016;254(12):2373-2385.

35. LJ O'Connor, IN Mistry, SL Collins et al. CYP450 Enzymes Effect Oxygen-Dependent Reduction of Azide-Based Fluorogenic Dyes. ACS Cent Sci. 2017;3:20-30.

36. W Feng, Y Wang, S Chen et al. 4-Nitroimidazole-3-hydroxyflavone conjugate as a fluorescent probe for hypoxic cells. Dyes and Pigments. 2016;131:145-153.

37. J Yuan, YQ Xu, NN Zhou et al. A highly selective turn-on fluorescent probe based on semi-cyanine for the detection of nitroreductase and hypoxic tumor cell imaging $R S C A d v$. 2014;4:56207-56210.

38. Zhao Li, Xiaohua Li, Xinghui Gao et al. Nitroreductase Detection and Hypoxic Tumor Cell Imaging by a Designed Sensitive and Selective Fluorescent Probe, 7-[(5-Nitrofuran-2-yl)methoxy]-3Hphenoxazin-3-one. Anal Chem. 2013;85:3926-3932.

39. Li D, Xu Y, Zhou N et al. A novel donor-two-acceptor type fluorophorebased probe for fast detection and intracellular imaging of nitroreductase. Dyes and Pigments. 2017;136:627-632. 
40. Hwan Myung Kim, Bong Rae Cho Small-Molecule Two-Photon Probes for Bioimaging Applications. Chem Rev. 2015;115:5014-5055.

41. Zhang J, HW Liu, XX Hu et al. Efficient Two-Photon Fluorescent Probe for Nitroreductase Detection and Hypoxia Imaging in Tumor Cells and Tissues. Anal Chem. 2015;87(23):11832-11839.

42. Guo T, Cui L, W Zhu et al. A highly sensitive long-wavelength fluorescence probe for nitroreductase and hypoxia: selective detection and quantification. Chem Commun. 2013;49(92):10820-10822.

43. Zhu D, Xue L, Li G, Jiang H A highly sensitive near-infrared ratiometric fluorescent probe for detecting nitroreductase and cellular imaging. Sensors and Actuators B. 2016;222:419-424.

44. Cui L, Shi Y, Zhang S et al. 2017 A NIR turn-on fluorescent probe applied in cytochrome $\mathrm{P} 450$ reductase detection and hypoxia imaging in tumor cells. Dyes and Pigments. 2016;139:587-592.

45. Hou TC, Wu YY, Chiang PY, et al. Near-infrared fluorescence activation probes based on disassembly-induced emission cyanine dye. Chem Sci. 2015;6(8):4643-4649.

46. Groves K, Bao K, Zhang J, et al. Synthesis and evaluation of nearinfrared fluorescent sulfonamide derivatives for imaging of hypoxiainduced carbonic anhydrase IX expression in tumors. Bioorg Med Chem Lett. 2012;22(1):653-657.

47. Wu JB, Shi C, G CY Chu, et al. Near-infrared fluorescence heptamethine carbocyanine dyes mediate imaging and targeted drug delivery for human brain tumor. Biomaterials. 2015;67:1-10.

48. Wu JB, Shao C, Li X, et al. Near-infrared fluorescence imaging of cancer mediated by tumor hypoxia and HIF1 $\alpha /$ OATPs signaling axis. Biomaterials. 2014;35(28):8175-8185.

49. Pan X, Wang X, Wang L, et al. Near-Infrared Fluorescence Probe for Monitoring the Metabolic Products of Vitamin C in HepG2 Cells under Normoxia and Hypoxia. Anal Chem0. 2015;87(14):7092-7097.

50. Hochstrasser M. Ubiquitin-dependent protein degradation. Annu Rev Genet. 1996;30:405-439.

51. Kuchimaru T, Suka T, Hirota K, et al. A novel injectable BRET-based in vivo imaging probe for detecting the activity of hypoxia-inducible factor regulated by the ubiquitin-proteasome system. Sci Rep. 2016;6:34311.

52. Busk M, Jakobsen S, Horsman MR, et al. PET imaging of tumor hypoxia using 18F-labeled pimonidazole. Acta Oncol. 2013;52(7):1300-1307.

53. Yang Y, XR Zhu, QG Xu, et al. Magnetic resonance imaging retinal oximetry: a quantitative physiological biomarker for early diabetic retinopathy? Diabetic Med. 2012;29(4):501-505.

54. Ross D, Beall HD, Siegel D, et al. Enzymology of bioreductive drug activation. Br J Cancer Suppl. 1996;27:1S-8S.

55. Uddin MI, Evans SM, Craft JR, et al. Applications of azo-based probes for imaging retinal hypoxia. ACS Med Chem Lett. 2015;6(4):445-449.

56. Evans SM. Molecular Probes for Imaging of Hypoxia in the Retina. Bioconjugate Chem. 2014;25:2030-2037.
57. Uddin MI, Evans SM, Craft JR, et al. In vivo Imaging of Retinal Hypoxia in a Model of Oxygen-Induced Retinopathy. Sci Rep. 2016;6(5):31011.

58. Dewhirst MW, Cao Y, Moeller B. Cycling hypoxia and free radicals regulate angiogenesis and radiotherapy response. Nat Rev Cancer. 2008;8(6):425-437.

59. Zhang $\mathrm{P}$, Huang $\mathrm{H}$, Chen $\mathrm{Y}$, et al. Ruthenium(II) anthraquinone complexes as two-photon luminescent probes for cycling hypoxia imaging in vivo. Biomaterials. 2015;53:522-531.

60. Zheng X, Wang X, Mao H, et al. Hypoxia-specific ultrasensitive detection of tumours and cancer cells in vivo. Nat Commun. 2015;6(5):5834.

61. Xiaoqian Zhang, Qi Zhao, Yanru Li, et al. Multifunctional Probe Based on Cationic Conjugated Polymers for Nitroreductase-Related Analysis: Sensing, Hypoxia Diagnosis, and Imaging. Anal Chem. 2017;89(10):5503-5510.

62. Madsen J, Canton I, Warren NJ, et al. Nile Blue-based nanosized pH sensors for simultaneous far-red and near-infrared live bioimaging. $J$ Am Chem Soc. 2013;135(39):14863-14870.

63. Joanna AM Braks, Linda Spiegelberg, Senada Koljenovic, et al. Optical Imaging of Tumor Response to Hyperbaric Oxygen Treatment and Irradiation in an Orthotopic Mouse Model of Head and Neck Squamous Cell Carcinoma. Mol Imaging Biol. 2015;17(5):633-642.

64. Liu L, Li B, Qin R, et al. Synthesis and characterization of new bifunctional nanocomposites possessing upconversion and oxygensensing properties. Nanotechnology. 2010;21(28):285701.

65. Jeong K, Park S, YD Lee, et al. Conjugated polymer/photochromophore binary nanococktails: bistable photoswitching of near-infrared fluorescence for in vivo imaging. Adv Mater. 2013;25(39):5574-5580.

66. Shamirian A, Afsari HS, Hassan A, et al. In vitro Detection of Hypoxia using a Ratiometric Quantum Dot-based Oxygen Sensor. ACS Sens. 2016;1(10):1244-1250.

67. Lv W, Yang T, Yu Q, et al. A Phosphorescent Iridium(III) ComplexModified Nanoprobe for Hypoxia Bioimaging Via Time-Resolved Luminescence Microscopy. Adv Sci. 2015;2(10):1500107.

68. Jianan Liu, Yong Liu, Wenbo $\mathrm{Bu}$ et al. Ultrasensitive Nanosensors Based on Upconversion Nanoparticles for Selective Hypoxia Imaging in vivo upon Near-Infrared Excitation. J Am Chem Soc. 2014;136(27):9701-9709.

69. Young Hun Seo, Min Ju Cho, Oug Jae Cheong et al. Low-bandgap biophotonic nanoblend: A platform for systemic disease targeting and functional imaging. Biomaterials. 2015;39:225-233.

70. Sevick Muraca EM Translation of near-infrared fluorescence imaging technologies: emerging clinical applications. Annu Rev Med. 2012;63:217-231.

71. Stanisavljevic M, Krizkova S, Vaculovicova M, Kizek R, Adam $\mathrm{V}$ Quantum dots-fluorescence resonance energy transferbased nanosensors and their application. Biosens Bioelectron. 2015;74:562-574. 